\title{
Application of Dose-Volume Histogram Prediction in Biologically Related Models for Nasopharyngeal Carcinomas Treatment Planning
}

\section{CURRENT STATUS: UNDER REVIEW}

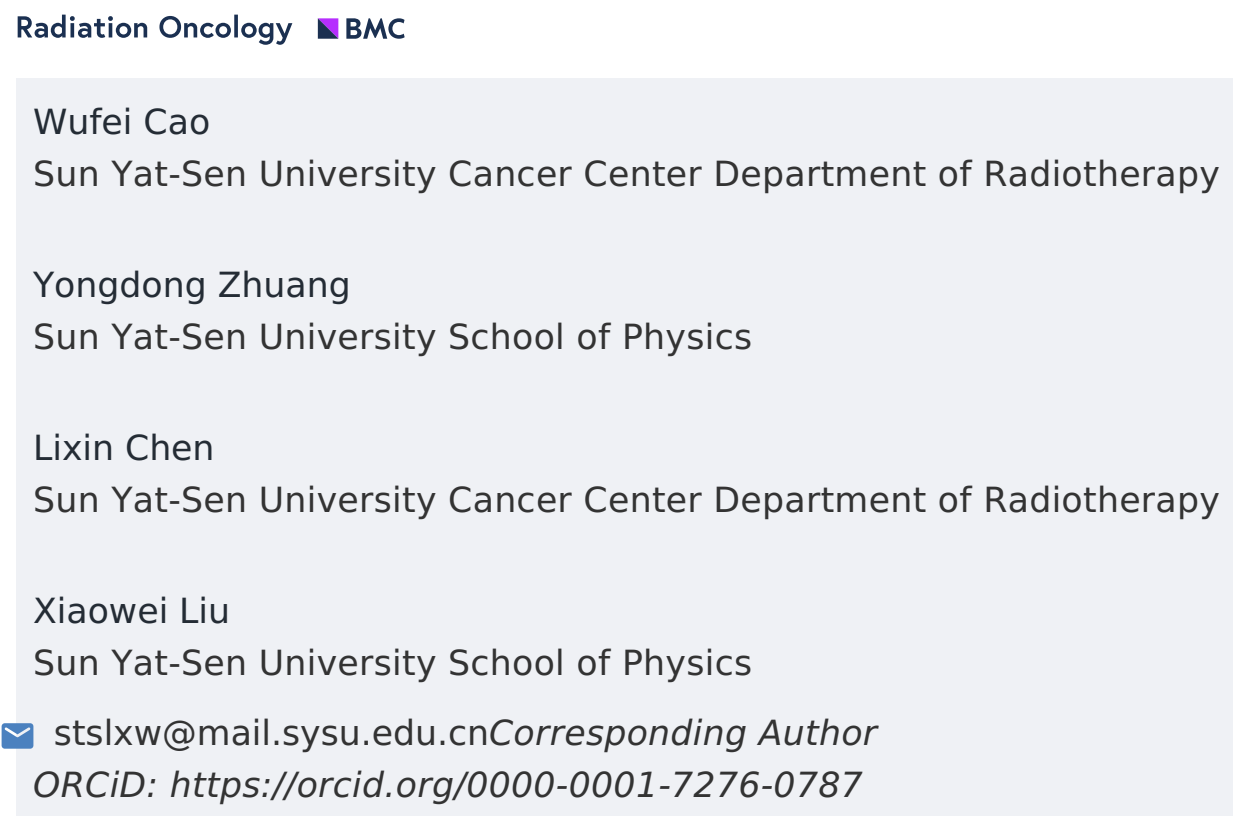

DOI:

10.21203/rs.3.rs-15981/v1

\section{SUBJECT AREAS}

Oncology Cancer Biology

KEYWORDS

DVH prediction, Biologically Related Models, nasopharyngeal carcinoma 
Abstract

Purpose: In this study, we employed a gated recurrent unit (GRU)-based recurrent neural network (RNN) to predict the dose-volume histogram (DVH) and investigated the feasibility and usefulness of this method in biologically related models for nasopharyngeal carcinomas treatment planning.

Methods and Materials: One hundred NPC patients were selected from a database containing clinical VMAT plans in recent two years; of these, the data from 80 patients were used to train the GRU-RNN, and the data of the other 20 patients were used for testing. For the prescribed doses of all the plans in 30 or 31 fractions, 70 Gy were delivered to PTV70 (the gross tumour volume with circumferential margin), 60 Gy were delivered to PTV60, 54 Gy were delivered to PTV54 and 66 Gy were delivered to PTV66 (lymph node gross tumour volume with circumferential margin). For each NPC patient, the DVHs of different organs at risk (OARs) were predicted by the trained GRU-based RNN using the information given by individual conformal beams. Based on the predictive DVHs, the equivalent uniform dose (EUD) were calculated, and directly applied as dose constrains in Treatment Planning optimization. The regenerated VMAT experimental plans (EPs) were evaluated by comparing them with the clinical plans (CPs).

Results: For the 20 test patients, the regenerated EPs guided by the GRU-RNN predictive model achieved very good consistency relative to the CPs. The EPs showed better dose sparing for many OARs and significant differences were found in the maximum/mean doses to the brain stem, brain stem PRV, spinal cord, lenses, temporal lobes and parotids with P-values $<0.05$, respectively. On average, compared with the CPs, the maximum/mean doses to these OARs were altered by $-3.44 \mathrm{~Gy}$, -1.94 Gy, -1.88 Gy, 0.44 Gy, 1.98 Gy and -1.82 Gy, respectively.

Conclusion: The GRU-RNN based DVH prediction method is capable of accurately and completely predicting the whole DVH for EUD calculation. The regenerated plans guided by the predictive EUD sparing were not inferior to the manual plans, indicating the great potential for improved planning and quality control in clinical applications.

Full Text

Due to technical limitations, full-text HTML conversion of this manuscript could not be completed. 
However, the manuscript can be downloaded and accessed as a PDF.

\section{Table}

Due to technical limitations, Table 1 is only available as a download in the supplementary files section.

Figures
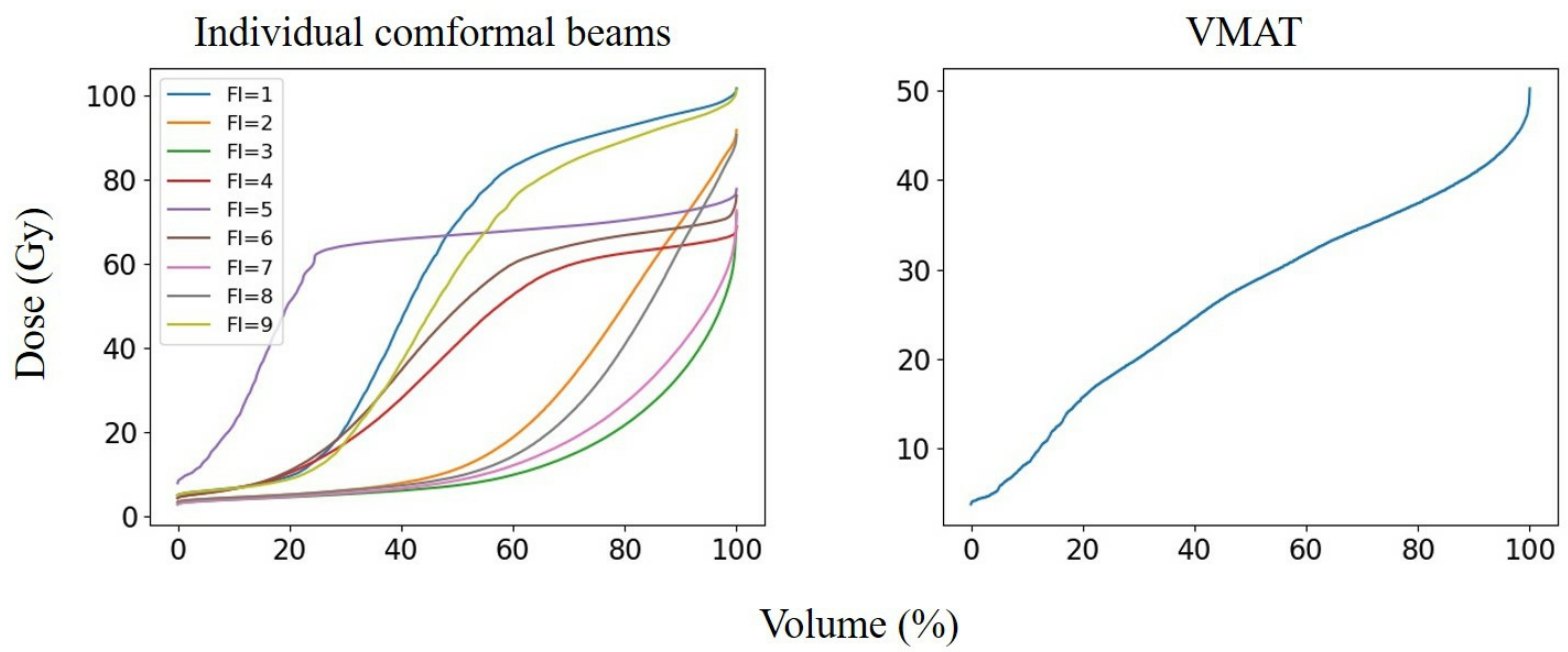

Figure 1

An example of the DVHs for a patient's brain stem, where $\mathrm{FI}=1,2,3, \ldots, 9$ denotes the fields corresponding to gantry angles of $160,120,80,40,0,320,280,240$, and 200 degrees, respectively. Dose (Gy) and Volume (\%) represent the delivered dose and percent OAR volume, respectively. 


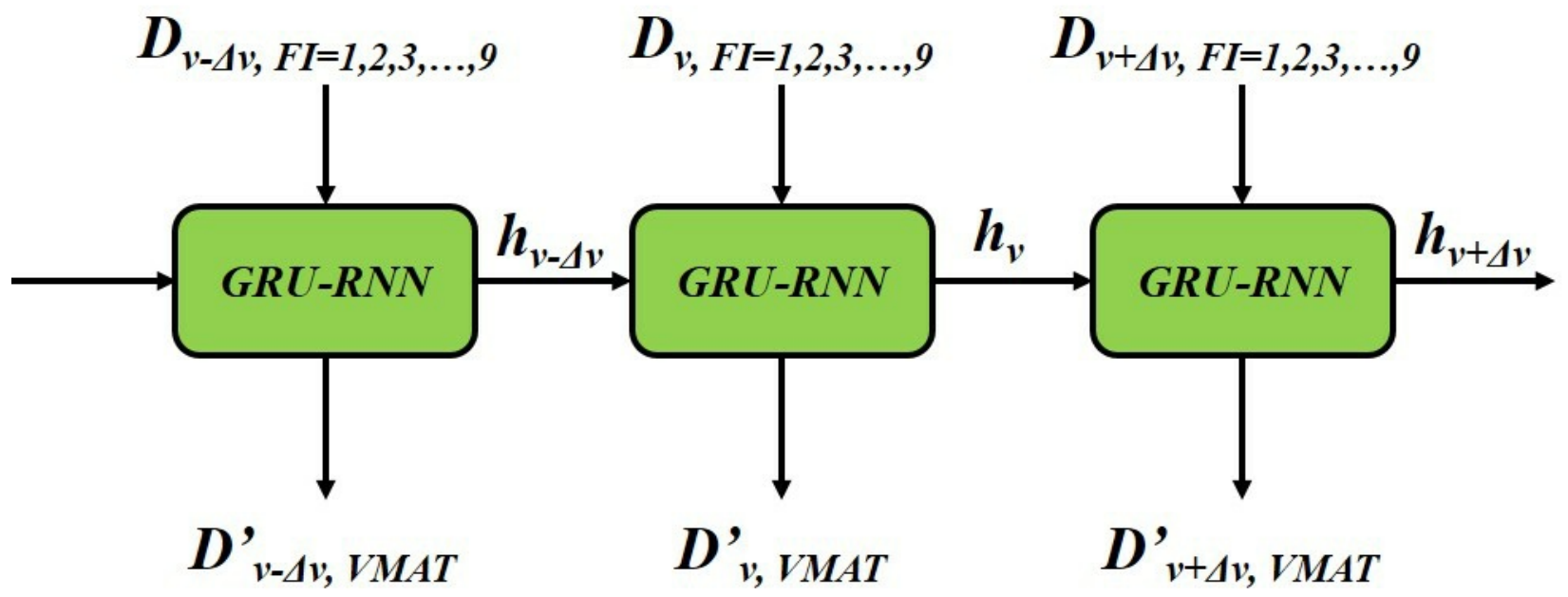

Figure 2

Flow chart of the GRU-RNN. RNN-GRU consists of 3 GRUs with the sizes of hidden states are 18, 9 and 1, respectively. D_v mean the volume proportion with deposition not greater than $\mathrm{D}$ is $\mathrm{v}$ and $\mathrm{D} \_\mathrm{v}^{\wedge}$ ' is the predictive value. In the practical, $\Delta \mathrm{v}$ was set to $0.1 \%$.

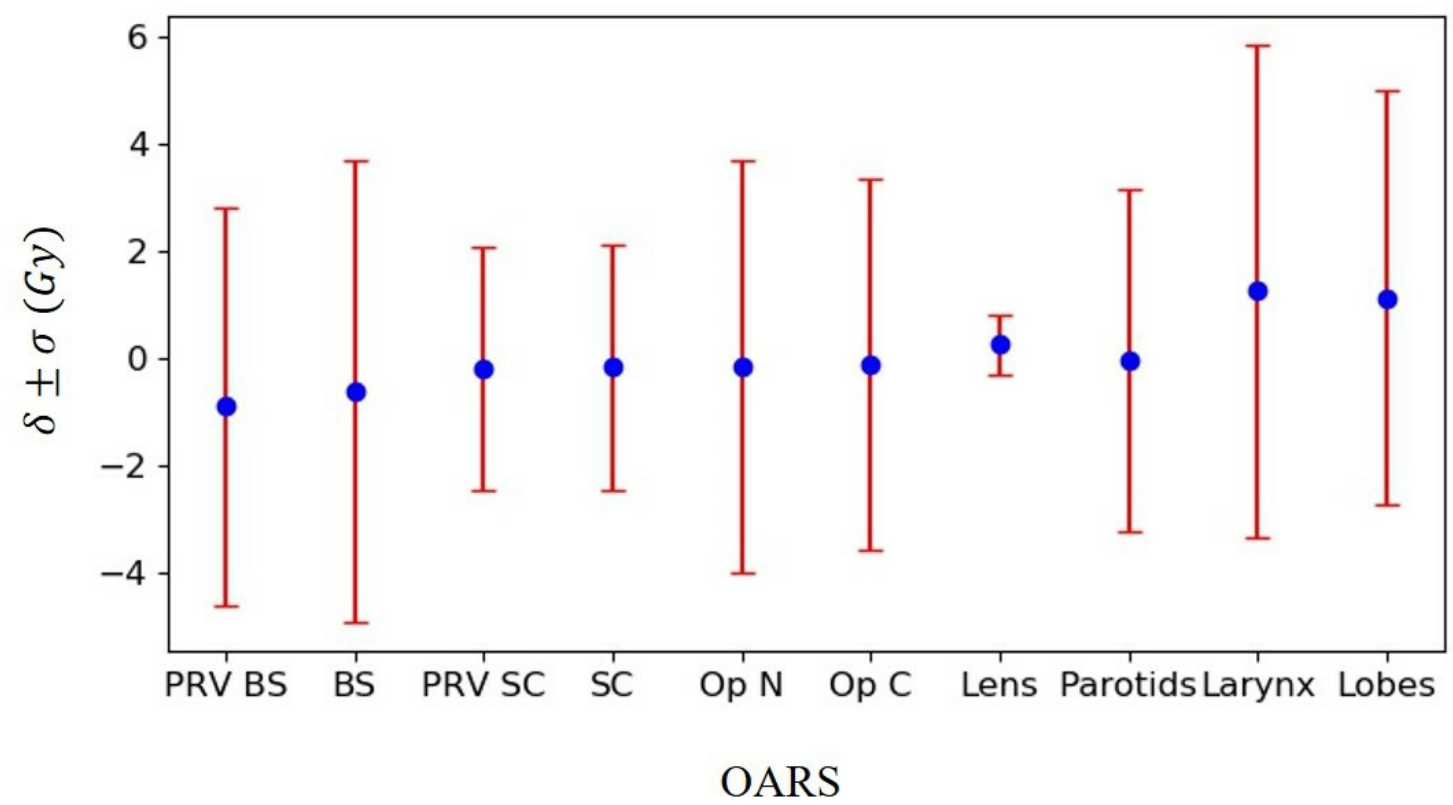

Figure 3

The $\delta$ and $\sigma$ presented the maximum dose prediction accuracy and precision of different OARs, including the brain stem (BS), spinal cord (SC), optic nerves (Op N), optic chiasm (Op C), lens, parotid glands (Parotids), larynx, and temporal lobes (Lobes). 

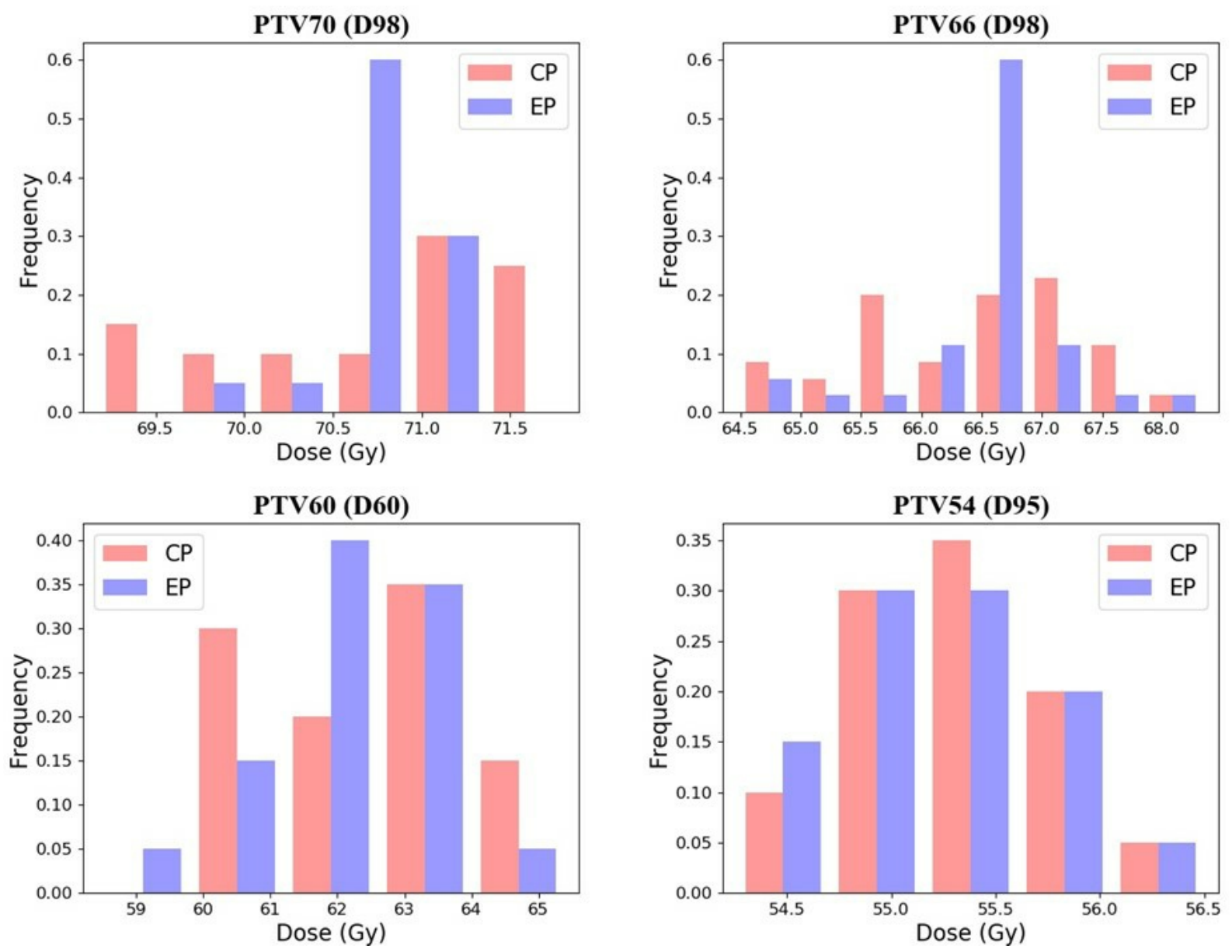

Figure 4

the distribution of D98 or D95 to the PTVs 

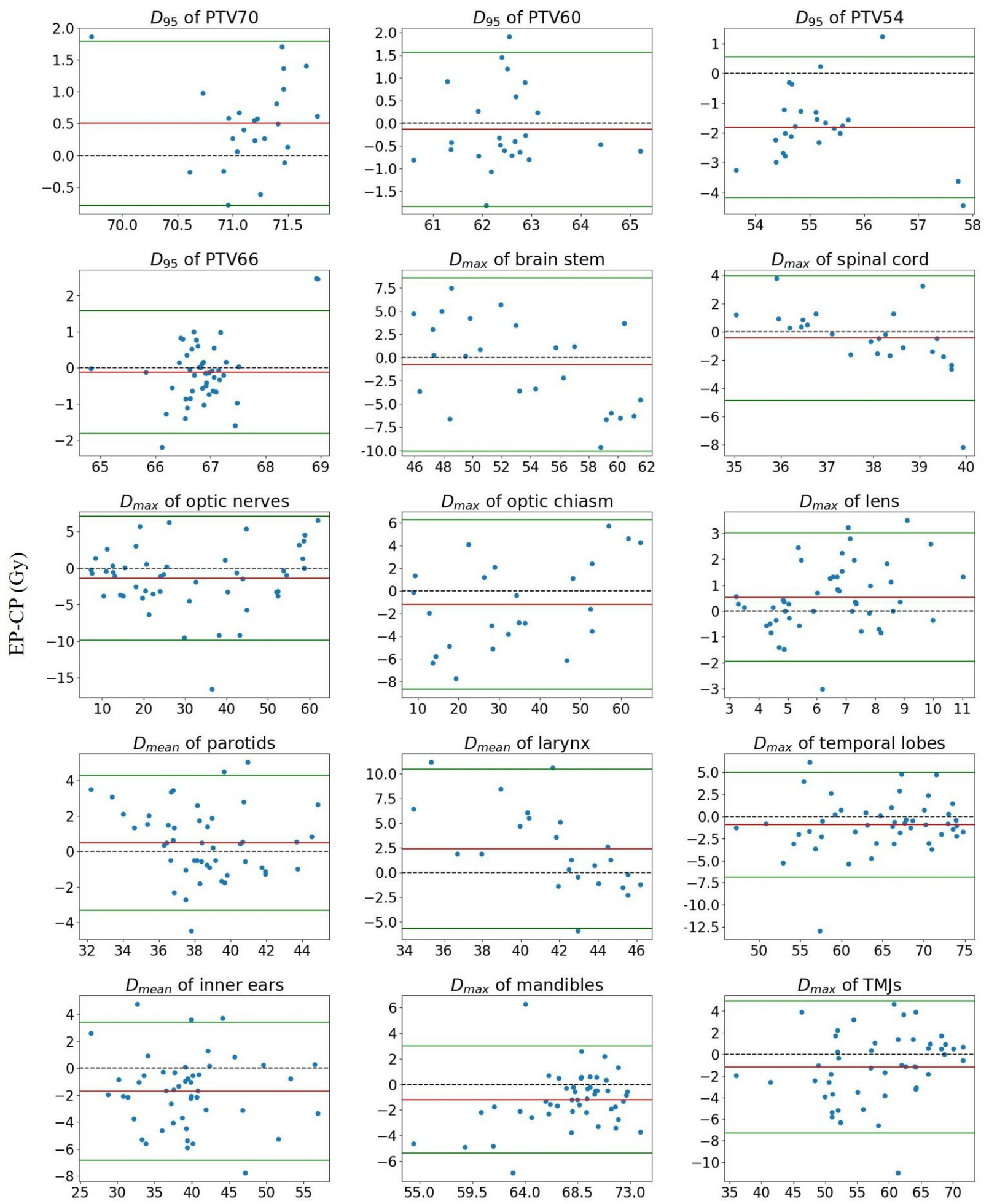

Mean of EP and CP (Gy)

Figure 5

Difference between CPS and EPs. Horizontal lines are drawn at the line of equality (black dashed line), the mean difference (red line), and the limits of agreement (green lines). The limits of agreement are defined as the mean difference \pm 1.96 SD of the differences. 


\section{Supplementary Files}

This is a list of supplementary files associated with this preprint. Click to download.

Table 1.PNG 